\title{
Inboard Stall Delay Due to Rotation
}

\author{
Horia Dumitrescu and Vladimir Cardoş \\ Institute of Statistical Mathematics and Applied Mathematics of the Romanian Academy \\ Romania
}

\section{Introduction}

In the design process of improved rotor blades the need for accurate aerodynamic predictions is very important. During the last years a large effort has gone into developing CFD tools for prediction of wind turbine flows (Duque et al., 2003; Fletcher et al., 2009; Sørensen et al.,2002). However, there are still some unclear aspects for engineers regarding the practical application of CFD, such as computational domain size, reference system for different computational blocks, mesh quality and mesh number, turbulence, etc. Thus, in the design process and in the power curve prediction of wind turbines, the aerodynamic forces are calculated with some form of the blade element method (BEM) and its extensions to the three-dimensional wing aerodynamics. The results obtained by the standard methods are reasonably accurate in the proximity of the design point, but in stalled condition the BEM is known to underpredict the forces acting on the blades (Himmelskamp, 1947). The major disadvantage of these methods is that the airflow is reduced to axial and circumferential flow components (Glauert, 1963). Disregarding radial flow components present in the bottom of separated boundary layers of rotating wings leads to alteration of lift and drag characteristics of the individual blade sections with respect to the 2-D airfoils (Bjorck, 1995).

Airfoil characteristics of lift $\left(C_{L}\right)$ and drag $\left(C_{D}\right)$ coefficients are normally derived from twodimensional (2-D) wind tunnel tests. However, after stall the flow over the inboard half of the rotor is strongly influenced by poorly understood 3-D effects (Banks \& Gadd, 1963; Tangler, 2002). The 3-D effects yield delayed stall with $C_{L}$ higher than 2.0 near the blade root location and with correspondingly high $C_{D}$. Now the design of constant speed, stallregulated wind turbines lacks adequate theory for predicting their peak and post-peak power and loads.

During the development of stall-regulated wind turbines, there were several attempts to predict 3-D post-stall airfoil characteristics (Corrigan \& Schlichting, 1994; Du \& Schling, 1998; Snel et al., 1993), but these methods predicted insufficient delayed stall in the root region and tended to extend the delayed stall region too far out on the blade.

The present work aims at giving a conceptualization of the complex 3-D flow field on a rotor blade, where stall begins and how it progresses, driven by the needs to formulate a reasonably simple model that complements the 2-D airfoil characteristics used to predict rotor performance.

Understanding wind turbine aerodynamics (Hansen \& Butterfield, 1993) in all working states is one of the key factors in making improved predictions of their performance. The flow field associated with wind turbines is highly three-dimensional and the transition to two-dimensional outboard separated flow is yet not well understood. A continued effort is 
necessary to improve the delayed stall modeling and bring it to a point where the prediction becomes acceptable. In the sequel, based on previous computed and measured results, a comprehensible model is devised to explain in physical terms the different phenomena that play a role and to clarify what can be modeled quantitatively in a scientific way and what is possible in an engineering environment.

In 1945 Himmelskamp (Himmelskamp, 1947) first described through measurements the 3-D and rotational effects on the boundary layer of a rotating propeller, finding lift coefficients much higher moving towards the rotation axis. Further experimental studies confirmed these early results, indicating in stall-delay and post-stalled higher lift coefficient values the main effects of rotation on wings. Measurements on wind turbine blades were performed by Ronstend (Ronsten, 1992), showing the differences between rotating and non-rotating pressure coefficients and aerodynamic loads, and by Tangler and Kocurek (Tangler \& Kocurek, 1993), who combined results from measurements with the classical BEM method to properly compute lift and drag coefficients and the rotor power in stalled conditions.

The theoretical foundations for the analysis of the rotational effects on rotating blades come at the late 40's with Sears (Sears, 1950), who derived a set of equations for the potential flow field around a cylindrical blade of infinite span in pure rotation. He stated that the spanwise component of velocity is dependent only upon the potential flow and it is independent of the span (the so-called independence principle). Then, Fogarty and Sears (Fogarty \& Sears, 1950) extended the former study to the potential flow around a rotating and advancing blade. They confirmed that, for a cylindrical blade advancing like a propeller, the tangential and axial velocity components are the same as in the 2-D motion at the local relative speed and incidence. A more comprehensive work was made once more by Fogarty (Fogarty, 1951), consisting of numerical computations on the laminar boundary layer of a rotating plate and blade with thickness. Here he showed that the separation line is unaffected by rotation and that the spanwise velocities in the boundary layer appeared small compared to the chordwise, and no large effects of rotation were observed in contrast to (Himmelskamp, 1947). A theoretical analysis done by Banks and Gadd (Banks \&Gadd, 1963), focused on demonstrating how rotation delays laminar separation. They found that the separation point is postponed due to rotation, and for extreme inboard stations the boundary layer is completely stabilized against separation.

In the NASA report done by McCroskey and Dwyer (McCroskey \& Dwyer, 1969), the socalled secondary effects in the laminar incompressible boundary layer of propeller and helicopter rotor blades are widely studied, by means of a combined, numerical and analytical approach. They showed that approaching the rotational axis, the Coriolis force in the cross flow direction becomes more important. On the other hand the centrifugal pumping effect is much weaker than was generally thought before, but its contribution increases particularly in the region of the separated flow. The last two decades have known the rising of computational fluid dynamics and the study of the boundary layer on rotating blade has often been carried on through a numerical approach. Sørensen (Sørensen, 1986) numerically solved the 3-D equations of the boundary layer on a rotating surface, using a viscous-inviscid interaction model. In his results the position of the separation line still appears the same as for 2-D predictions, but where separations are more pronounced a larger difference between the lift coefficient calculated for the 2-D and 3-D case is noticed. A quasi 3-D approach, based on the viscous-inviscid interaction method, was introduced by Snel et al (Snel et al., 1993) and results were compared with measurements. They proposed a semi-empirical law for the correction of the 2-D lift curve, identifying the local chord to radii 
$(c / r)$ ratio of the blade section as the main parameter of influence. This result has been confirmed by Shen and Sørensen (Shen \& Sørensen, 1999), and by Chaviaropoulos and Hansen (Chaviaropoulos \& Hansen, 2000), who performed airfoil computations applying a quasi 3-D Navier-Stokes model, based on the streamfunction-vorticity formulation and respectively a primitive variables form. Du and Seling (Du \& Selling, 2000), and Dumitrescu and Cardoş (Dumitrescu \& Cardoş, 2010), investigated the effects of rotation on blade boundary layers by solving the 3-D integral boundary-layer equations with the assumed velocity profiles and a closure model. Dumitrescu and Cardoss studying the boundary layer behavior very close to the rotation center $(r / c<1)$ stated that the stall delay depends strongly on the leading edge separation bubbles formed on inboard blade segment due to a suction effect at the root area of the blade (Dumitrescu \& Cardoş, 2009).

Recently, with the advent of the supercomputer, computational fluid dynamics (CFD) tools have been employed to investigate the stall-delay for wind turbines (Duque et al., 2003; Fletcher et al., 2009; Sørensen et al., 2002). These calculations in addition to Narramore and Vermeland's results (Narramore \& Vermeland, 1992) showed that the 3-D rotational effect was particularly pronounced for the inboard sections, but the genesis of this phenomenon is a problem still open. The present concern aims at giving explanations in physics terms of the different features widely-observed experimentally and computationally in wind turbine flow.

\section{Flow at low wind speeds}

At low wind speed conditions, i.e. at high speed ratios (TSR $>3$ ), the visualization of the computed flow indicates that the flow is well-behaved and attached over much of the rotor, Fig. 1. Figure 1 shows the separated area and radial flow on the suction side of a commercial blade with $40 \mathrm{~m}$ length at the design tip speed ratio $(\mathrm{TSR}=5)$; the secondary flow is strongest at approximately $0.17 R$ and reaches up to $0.31 R$, where $R$ is the rotor radius. The local air velocity relative to a rotor blade consists of free-wind velocity $V_{w}$ defined as the wind speed if there were no rotor present, that due to the blade motion $\Omega_{b} r$ and the wake induced velocities; at high TSR, a weak wake (Glauert, 1963) occurs and its rotational induction velocity can be neglected. Wind turbine blade sections can operate in two main flow regimes depending on the size of the rotation parameter defined as the ratio of wind velocity to the local tangential velocity $V_{w} / \Omega_{b} r$. If the rotation parameter is less than unity along the entire span, and for properly twisted blades, the flow is mostly two-dimensional and attached, while for rotation parameter greater than unity the flow is neither twodimensional nor steady, and is strongly affected by rotation. At high tip speed ratios the subunitary $V_{w} / \Omega_{b} r$ condition is accomplished and the blade sections usually operate at prestall incidences. Then, the boundary layer is attached all the way to the trailing edge on the outside blade and is separated at trailing edge only on the inside blade, Fig. 1 . At the root area of the blade the flow close to the hub behaves like a rotating disk in a fluid at rest (Fig. 2a), where the centrifugal forces induce a spinning motion in the separated flow; and a radial velocity field is more or less uniform (Fig. $2 b$ ). Beginning at the hub, this secondary flow generates the so-called centrifugal pumping mechanism, acting in separated trailing edge flow. On the other hand, the Coriolis force acts in the chordwise direction as a favorable pressure that mitigates separation at the trailing edge along the whole span. 


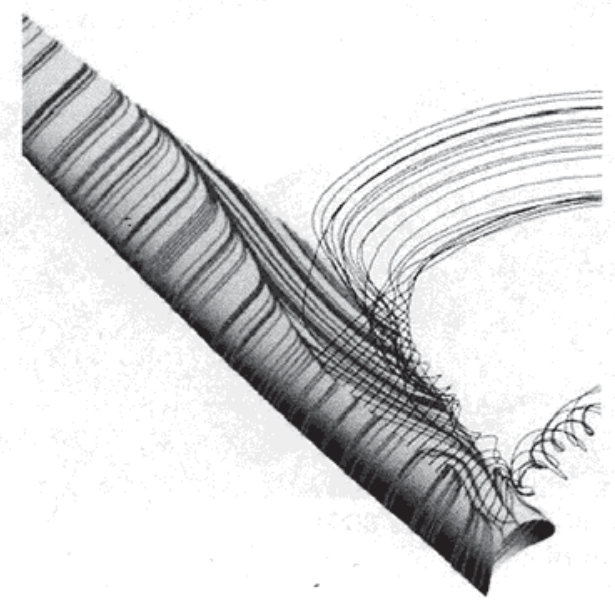

Fig. 1. Typical surface and 3-D streamlines on the blade suction side at low wind speeds $(\mathrm{TSR}=5)$
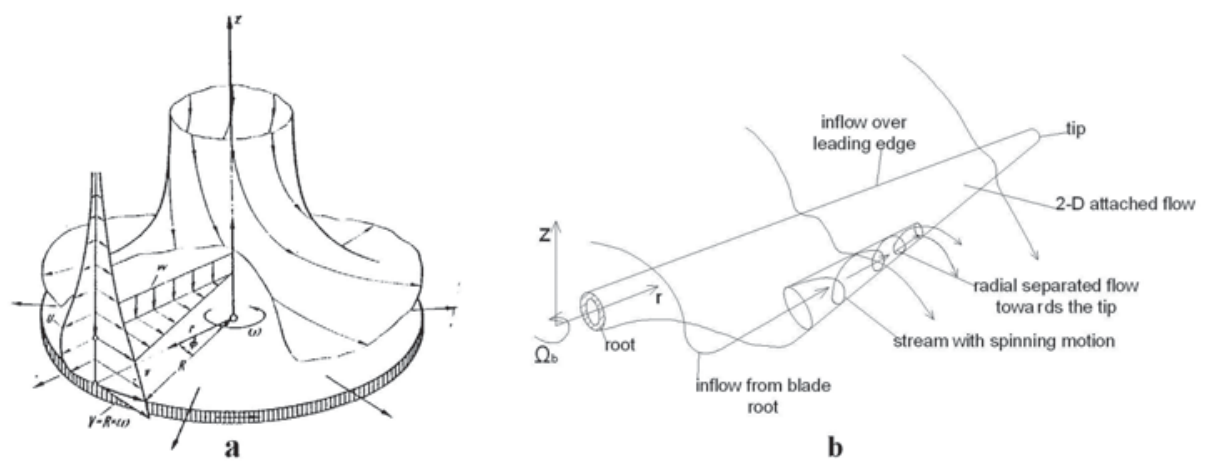

Fig. 2. Pumping-work mode of a wind turbine at low wind speeds (TSR >3.0): a) conceptof flow close to a rotating disk in a fluid at rest; b) model of the separation flow (Corten, 2001).

Therefore, at low wind speed, the main rotational effect is due to the Coriolis force which delays the occurrence of separation to a point further downstream towards the trailing edge, and by this the suction pressures move towards higher levels as $r / c$ decreases. The pumping effect is much weaker than was generally thought before.

The pressure field created by the presence of the turbine is related to the incoming flow field around the blade, taken as being composed of the free wind velocity and the so-called induction velocity due to the rotor and its wake. Thus, the incoming field results from a weak interaction between two different flows: one axial and the other rotational $\left(V_{w} / \Omega_{b} r<1\right)$. In such a weak interaction flow, the basic assumptions made are: 
- the radial independence principle is applied to flow effects, i.e. induction velocities used at a certain radial station depend only on the local aerodynamic forces at that same station;

- the mathematical description of the air flow over the blades is based on the 2-D flow potential independent of the span, and on corrections for viscosity and 3-D rotational effects.

These assumptions suitable to BEM methods reduce the complexity of the problem by an order of magnitude yielding reliable results for the local forces and the overall torque in the proximity of the design point, at high tip speed ratios. In order to estimate the 3-D rotational effects, usually neglected in the traditional BEM model, the flow around a hypothetic blade with prestall/stall incidence and chord constant along the whole span is considered in the sequel.

\subsection{Representation of flow elements}

The set of equations including a simplified form of the inviscid flow and the full threedimensional boundary-layer equations are used to identify the influence of the3-D rotational effects at low wind speeds.

A. Inviscid flow. In order to find the velocity at the airfoil surface in absence of viscous effects, the reference velocity at a point on a rotating wind turbine blade is

$$
U_{r}=V_{w} \sqrt{\left(\lambda \frac{r}{R}\right)^{2}+(1-a)^{2}}
$$

where $V_{w}$ is the wind speed, $\lambda=\frac{\Omega_{b} R}{V_{w}}$ is the tip-speed ratio (TSR), $R$ is the radius of the turbine and $a$ is the axial induced velocity interference, a function of the speed ratio $\lambda$ (Burton et al., 2001). Starting from the idea of Fogarty and Sears (Fogarty \& Sears, 1950), an inviscid edge velocity can be calculated as

$$
U=\Omega_{b} r \frac{\partial \phi}{\partial \theta}, V=\Omega_{b}(\phi-2 \theta), W=\Omega_{b} r \frac{\partial \phi}{\partial z}
$$

where $U, V$ and $W$ represent the velocity components in the cylindrical coordinate system $(\theta, r, z)$, which rotates with the blade with a constant-rotational speed $\Omega_{b}$ (Fig. 3). $\phi=\phi(\theta, z)$ denotes the 2-D potential solution, that is constant at all radial positions. The interesting point regarding this set of equations (2) is that the spanwise component $V$ can be derived from the local 2-D velocity potential. However, this spanwise component is very small and thus neglected in the present work. The potential edge velocity components can be approached as

$$
U_{e}=U_{r} U_{2 D}, V=0
$$

where the non-dimensional velocity $U_{2 D}$ could be obtained by a viscous-inviscid interaction procedure for flow past a 2-D airfoil (Drela, 1989). Since the primary concern of the present work is to investigate the rotational 3-D effects on prestalled and stalled blades by means of the boundary layer method, the inviscid pressure distribution is simply considered as 

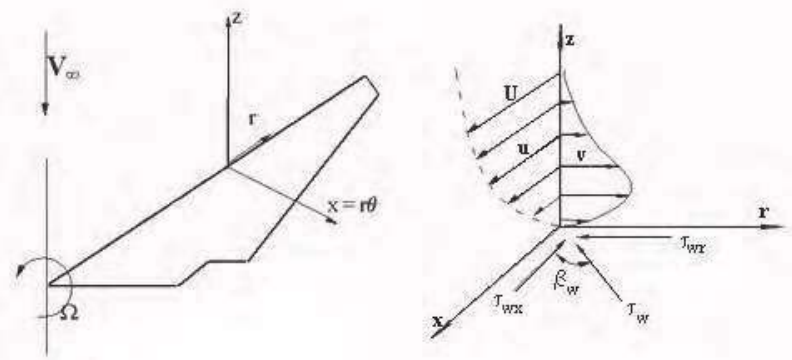

Fig. 3. Cylindrical coordinate system and notation used.

$$
C_{p U}=\left|C_{p m}\right|\left(\sqrt{\frac{x}{c}\left(2-\frac{x}{c}\right)}-1\right), C_{p L}=1-\sqrt{\frac{x}{c}\left(2-\frac{x}{c}\right)}
$$

where $C_{p}=\frac{p-p_{\infty}}{\rho / 2\left(V_{w}^{2}+\Omega_{b} r^{2}\right)}$ is the local pressure coefficient, $x / c$ is the airfoil abscissa by airfoil chord and subscripts $U$ and $L$ indicate properties on the upper surface and lower surface respectively. The scale parameter $\left|C_{p m}\right|$ is equal to maximum value of $C_{p}$ and is only dependent on the local aerodynamic field: the airfoil shape, incidence and Reynolds number. This one-parameter pressure distribution family ranges a large gamut from the prestall distributions, values of $\left|C_{p m}\right|=2-6$, until the stall and post-stall distributions with $\left|C_{p m}\right|=7-12$. Examples of distributions and experimental comparisons are shown in Fig. 4 .
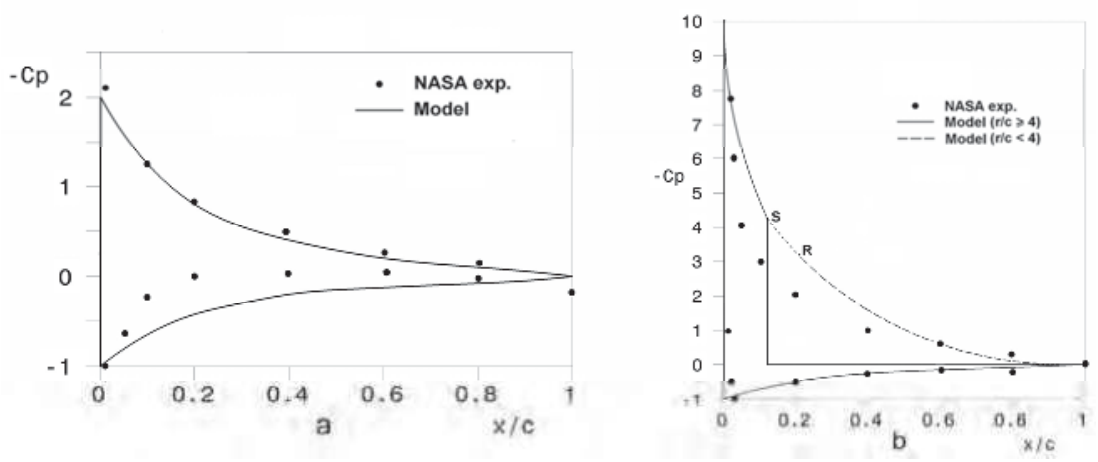

Fig. 4. Pressure distributions compared to experimental data for a NACA 0012 airfoil:

a) $\alpha=6.75 \operatorname{deg}\left(\left|C_{p m}\right|=2\right)$; b) $\alpha=19.35 \operatorname{deg}\left(\left|C_{p m}\right|=9\right)$

Because a wind turbine can operate a long time at low tip speed ratios, the inboard regions of the blade are stalled, and there leading-edge separation bubbles can occur (Dumitrescu \& Cardoş, 2010). Behind the mild separation it is assumed that the flow relaxes with the vanishing skin friction. Therefore, the following flow is applied to solve 


$$
\begin{gathered}
U_{e}=U_{r} \sqrt{1-C_{p}}, \text { for } \frac{x}{c}<\left(\frac{x}{c}\right)_{s e p} \\
U_{e} \delta_{2 x}^{1 /(H+2)}=\text { const., for } \frac{x}{c} \geq\left(\frac{x}{c}\right)_{s e p}
\end{gathered}
$$

where $\delta_{2 x}$ and $H$ are, respectively, the momentum thickness and the boundary-layer shape parameter in the streamwise direction.

Figures 5 and 6 illustrate such chordwise inviscid velocity distributions and the corresponding variations of the peripheral skin-friction coefficient $C_{f x}$ and the boundary layer shape parameter $H$, calculated for various values of $\left|C_{p m}\right|$ (a velocity gradient like parameter) and $r / c=\infty(2-D)$. Laminar separation takes place at $C_{f x}=0$, and a given value of the shape factor, $H=3.4$, is used as the criterion for turbulent separation/reattachment (Cebeci \& Cousteix, 1999). The "transition point" $x_{t}$ is the point that corresponds to the minimum skin-friction, which sometimes can be the point of laminar separation.

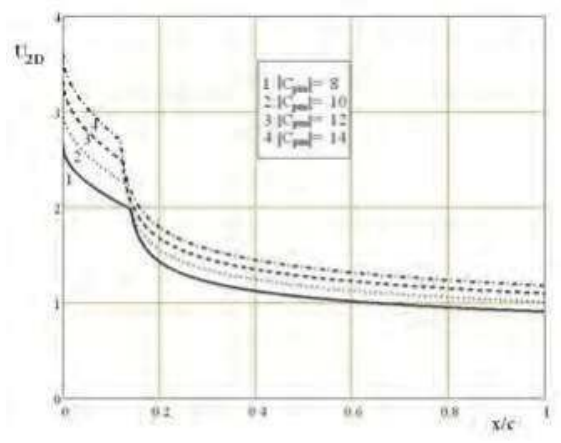

Fig. 5. Chordwise inviscid velocity distribution for various velocity gradient parameters $\left|C_{p m}\right|$
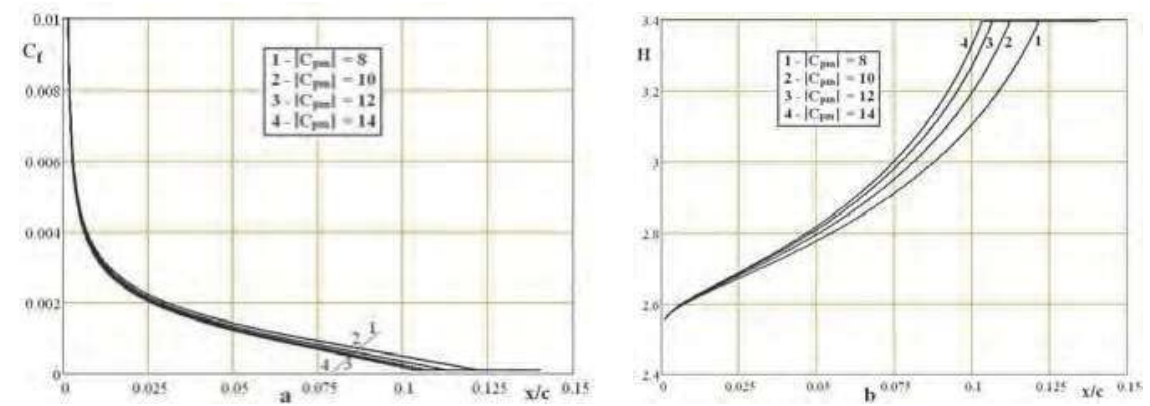

Fig. 6. Variation of a) the peripheral skin-friction coefficient; b) the boundary-layer shape parameter. 


\section{B. Viscous flow}

Momentum integral equations. The flow in the boundary layer on a rotating blade in attached as well as in stalled conditions is represented using the integral formulation developed in (Dumitrescu \& Cardoş, 2010) for analyzing separated and reattaching turbulent flows involving leading-edge separation bubbles. The 3-D incompressible steady boundary layer equations are written in the cylindrical coordinate system $(\theta, r, z)$ which rotate with the blade at a constant rotational speed $\Omega_{z}=\Omega_{b}$ (Fig. 3); $\theta$ denotes the peripheral, $z$ the axial and $r$ the radial (spanwise) direction. The equations used early (Cebeci \& Cousteix, 1999) are shown in their conservative laminar form:

Continuity

$$
\frac{\partial u}{\partial \theta}+\frac{\partial(r v)}{\partial r}+\frac{\partial(r w)}{\partial z}=0
$$

Momentum, $\theta$ component:

$$
\frac{\partial\left(u^{2}\right)}{r \partial \theta}+\frac{\partial(u v)}{\partial r}+\frac{\partial(u w)}{\partial z}+\frac{2 v}{r}\left(u-\Omega_{z} r\right)=-\frac{1}{\rho} \frac{\partial P}{r \partial \theta}+\frac{1}{\rho} \frac{\partial \tau_{x}}{\partial z}, \frac{1}{\rho} \frac{\partial \tau_{x}}{\partial z}=v \frac{\partial^{2} u}{\partial z^{2}},
$$

Momentum, $r$ component:

$$
\frac{\partial(u v)}{r \partial \theta}+\frac{\partial\left(v^{2}\right)}{\partial r}+\frac{\partial(v w)}{\partial z}+\frac{v^{2}}{r}-\frac{u}{r}\left(u-2 \Omega_{z} r\right)=-\frac{1}{\rho} \frac{\partial P}{\partial r}+\frac{1}{\rho} \frac{\partial \tau_{r}}{\partial z}, \quad \frac{1}{\rho} \frac{\partial \tau_{r}}{\partial z}=v \frac{\partial^{2} v}{\partial z^{2}},
$$

where $u, v$ and $w$ stand for velocity components in $\theta, r$ and $z$ directions, respectively, $r$ for the local radius measured from the centre of rotation, $\rho$ for the fluid density, $v$ for the kinematic viscosity, and $P$ is a pressure like term including the centrifugal effect

$$
P=\frac{p}{\rho}-\frac{1}{2}\left(\Omega_{z} r\right)^{2}
$$

with $p$ denoting the static pressure.

The equations (7)-(9) are integrated with respect to $z$ (normal to blade) from 0 to $\delta$ (boundary layer thickness) and the integral forms of equations are obtained as

$$
\begin{gathered}
\frac{\partial \delta_{2 x}}{\partial x}+\frac{\partial \delta_{2 x r}}{\partial r}+\frac{1}{U_{e}} \frac{\partial U_{e}}{\partial x}\left(2 \delta_{2 x}+\delta_{1 x}\right)-\frac{\zeta}{U e}\left(2 \delta_{2 x r}+\delta_{1 r}\right)-2 \frac{\Omega_{z}}{U_{e}} \delta_{1 r}=\frac{\tau_{w x}}{\rho U_{e}^{2}} \\
\frac{\partial\left(\delta_{2 x r}+\delta_{1 r}\right)}{\partial x}+\frac{\partial \delta_{2 r}}{\partial r}+\frac{2}{U_{e}} \frac{\partial U_{e}}{\partial x}\left(\delta_{2 x r}+\delta_{1 r}\right)+\frac{1}{U_{e}} \frac{\partial U_{e}}{\partial r}\left(\delta_{2 r}+\delta_{1 x}+\delta_{2 x}\right)+2 \frac{\Omega_{z}}{U_{e}} \delta_{1 x}- \\
-\frac{\zeta}{U_{e}}\left(\delta_{2 r}-\delta_{1 x}-\delta_{2 x}\right)=\frac{\tau_{w r}}{\rho U_{e}^{2}}
\end{gathered}
$$

where $\left(U_{e}, V_{e}=0\right)$ are the inviscid freestream velocity components and $\left(C_{f x}, C_{f r}\right)$ are the skinfriction coefficient components. The various boundary layer thicknesses are defined as 


$$
\begin{aligned}
& \delta_{1 x}=\int_{0}^{\delta}\left(1-\frac{u}{U_{e}}\right) \mathrm{d} z, \quad \delta_{1 r}=-\int_{0}^{\delta} \frac{v}{U_{e}} \mathrm{~d} z, \\
& \delta_{2 x}=\int_{0}^{\delta}\left(1-\frac{u}{U_{e}}\right) \frac{u}{U_{e}} \mathrm{~d} z, \quad \delta_{2 r}=-\int_{0}^{\delta} \frac{v^{2}}{U_{e}^{2}} \mathrm{~d} z, \quad \delta_{2 x r}=\int_{0}^{\delta}\left(1-\frac{u}{U_{e}}\right) \frac{v}{U_{e}} \mathrm{~d} z,
\end{aligned}
$$

An order of magnitude analysis shows that $\frac{\partial \delta_{2 x r}}{\partial r}=O\left(\varepsilon \delta_{2 x}\right)$ and $\frac{\partial \delta_{2 r}}{\partial r}=O\left(\varepsilon^{2} \delta_{2 x}\right)$, and thereby these terms can be neglected as a first approximation. The widely used Pohlhausen (Schlichting, 1979) and Mager (Mager, 1951) velocity profiles and the associated closure relations are introduced to solve the laminar integral boundary layer equations (Dumitrescu et al., 2007). For the turbulent boundary layer a power law-type of velocity profile is assumed for the mean stream velocity profile

$$
\frac{u}{U_{e}}=\left(\frac{z}{\delta}\right)^{(H-1) / 2}
$$

where $H$ is the local boundary layer shape factor.

The Mager cross flow profile is assumed (Mager, 1951)

$$
\frac{v}{u}=\varepsilon_{w}\left(1-\frac{z}{\delta}\right)^{2},
$$

where $\varepsilon_{w}$ is the limiting streamline parameter $\left(\tan \beta_{w}\right)$.

Equations (11) and (12) can now be written in terms of the parameters $\delta_{2 x}, \varepsilon_{w}, H$ and $C_{f x}$,

$$
\begin{gathered}
\frac{\partial \delta_{2 x}}{\partial x}+(2+H) \delta_{2 x} \frac{1}{U_{e}} \frac{\partial U_{e}}{\partial x}+\frac{\partial}{\partial r}\left(L \varepsilon_{w} \delta_{2 x}\right)-\frac{\zeta}{U_{e}}(2 L+M) \varepsilon_{w} \delta_{2 x}-2 \frac{\Omega_{z}}{U_{e}} M \varepsilon_{w} \delta_{2 x}=\frac{1}{2} C_{f x} \\
\frac{\partial}{\partial x}\left[(L+M) \varepsilon_{w} \delta_{2 x}\right]+\frac{2}{U_{e}} \frac{\partial U_{e}}{\partial x}(L+M) \varepsilon_{w} \delta_{2 x}+\frac{\partial}{\partial r}\left(N \varepsilon_{w}^{2} \delta_{2 x}\right)+\frac{1}{U_{e}} \frac{\partial U_{e}}{\partial r}\left(N \varepsilon_{w}^{2}+H+1\right) \delta_{2 x}- \\
-\frac{\zeta}{U_{e}}\left(N \varepsilon_{w}^{2}-H-1\right) \delta_{2 x}+2 \frac{\Omega_{z}}{U_{e}} H \delta_{2 x}=\frac{1}{2} C_{f x} \varepsilon_{w}, \\
L=\frac{\delta_{2 x r}}{\varepsilon_{w} \delta_{2 x}}=\frac{2(7 H+15)}{(H+2)(H+3)(H+5)}, \\
M=\frac{\delta_{1 r}}{\varepsilon_{w} \delta_{2 x}}=-\frac{16 H}{(H-1)(H+3)(H+5)} \\
N=\frac{\delta_{2 r}}{\varepsilon_{w}^{2} \delta_{2 x}}=-\frac{24}{(H-1)(H+2)(H+3)(H+4)} .
\end{gathered}
$$

The skin-friction relation for flows with pressure gradients and rotation effects is based on the experimental data for a turbulent boundary layer in a rotating channel (Lakshminarayana \& Govindan, 1981), 


$$
C_{f x}=0.172 \operatorname{Re}_{\delta_{2 x}}^{-0.268} 10^{-0.678 H}\left(1+B_{1} \sqrt{\varepsilon_{w}\left(x-x_{t}\right) / c}\right)
$$

This correlation is a modified version of the correlation developed by Ludwieg and Tillmann (Ludwieg \& Tillman, 1949), which includes the effect of rotation. In this relation $B_{1}$ is an empiric constant (a value of 0.52 is used), $\operatorname{Re}_{\delta_{2 x}}$ is the Reynolds number, based on the streamwise velocity at the edge of the boundary layer and the streamwise momentum thickness $\delta_{2 x}$, and $x_{t}$ is the distance between the leading edge and the transition point along the streamwise direction (the laminar separation point is used).

\section{Closure model-entrainment}

The pressure gradients cause large changes in velocity profiles and, consequently, in the shape parameter $H$. The variation of $H$ cannot be neglected and an additional equation is required. Out of the available auxiliary equations, only the energy integral equation and the entrainment equation have been suitable for the turbulent boundary layers.

The entrainment equation is chosen to model the rotor boundary layer, which in the coordinate system used here can be written as

$$
\frac{\partial\left(\delta-\delta_{1 x}\right)}{\partial x}+\left(\delta-\delta_{1}\right) \frac{1}{U_{e}} \frac{\partial U_{e}}{\partial x}-\frac{\partial \delta_{1 r}}{\partial r}+\frac{\zeta}{U_{e}} \delta_{r}=C_{E}
$$

where the entrainment coefficient $C_{E} \equiv\left(\frac{\partial \delta}{\partial x}-\frac{W_{e}}{U_{e}}\right)$ is a function of the factor $H_{1}=\left(\delta-\delta_{1 x}\right) / \delta_{2 x} \cdot C_{E}$ represents the volume flow rate per unit area through the surface $\delta(x, r)$ and is the rate of entrainment of inviscid external flow into the boundary layer.

The entrainment function $C_{E}$ for 3-D flow is not yet available and hence, the correlation for 2-D flow is used (Head, 1958):

$$
C_{E}=0.0306\left(H_{1}-3.0\right)^{-0.653}
$$

Also, the similarity solutions have shown that $H_{1}$ is a function of the streamwise boundarylayer shape parameter $H$. This relationship, which results from a best fit to experimental data (Lock \& Williams, 1987) is

$$
H_{1}=2+1.5\left(\frac{1.12}{H-1}\right)^{1.093}+0.5\left(\frac{H-1}{1.12}\right)^{1.093}, \text { for } H<4 .
$$

Then, it is assumed that the variation of the entrainment rate with $H_{1}$ follows the same relationship for three-dimensional flows.

Equation (19) is written in a form similar to Eqs. (15) and (16)

$$
\frac{\partial}{\partial x}\left(\delta_{2 x} H_{1}\right)+\left(\delta_{2 x} H_{1}\right) \frac{1}{U_{e}} \frac{\partial U_{e}}{\partial x}-\frac{\partial}{\partial r}\left(M \varepsilon_{w} \delta_{2 x}\right)+\left(M \varepsilon_{w} \delta_{2 x}\right) \frac{\zeta}{U_{e}}=C_{E}\left(H_{1}\right)
$$

Equations (15), (16) and (22) are to be solved for $\delta_{2 x}, \varepsilon_{w}$, and $H\left(H_{1}\right.$ is related to $H$, Eq. (21)) with the prescribed boundary conditions. At the leading edge, $\delta_{2 x}$ and $\varepsilon_{w}$ are assumed to be zero, and an initial value of 2.55 (laminar flow) is assumed for $H$. 


\subsection{Application}

A hypothetic blade, with constant incidence and chord along the span is chosen to analyze the rotational effects on the boundary layer of a wind turbine blade. In this example, an external flow with the distribution of velocity on the surface of blade given by Eqs. (5) and (6) is imposed.

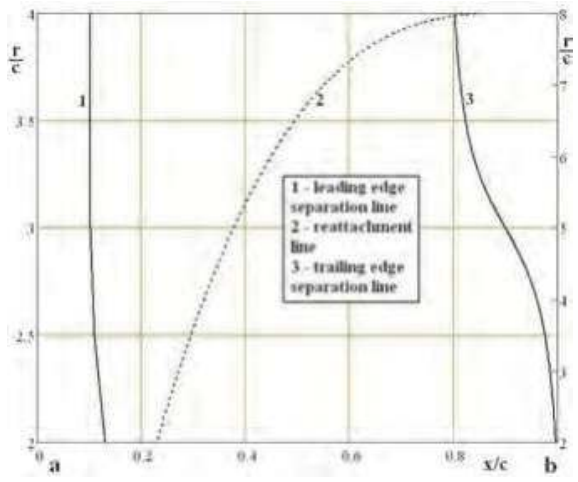

Fig. 7. Relationship between the separation points and the blade radius: a) at stall incidence; b) at prestall incidence.

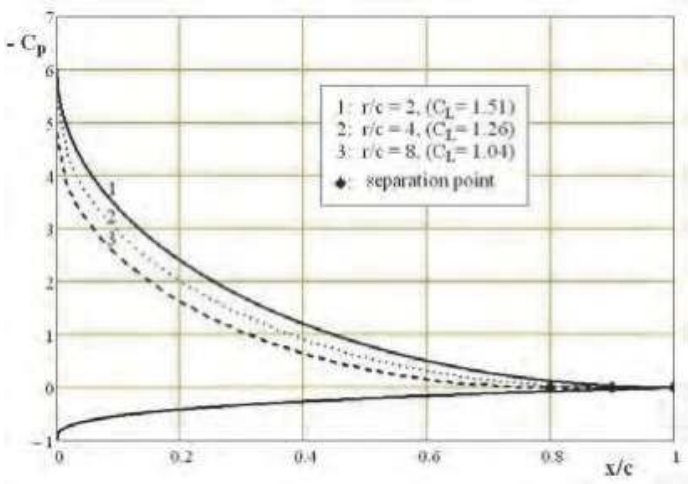

Fig. 8. Influence of the $\mathrm{r} / \mathrm{c}$ ratio on the suction pressure coefficient and lift coefficient at prestall incidence, $\left|C_{p m}\right|=6(\alpha \sim 14 \mathrm{deg})$.

The curves plotted in Fig. 7 are calculated on the prestall $\left(\left|C_{p m}\right|=6\right)$ and stall $\left(\left|C_{p m}\right|=10\right)$ conditions, with a Reynolds number of $10^{6}$. Relationships between the separation points and the blade radius are shown in Fig. 7, in which two apparent trends can be seen. Firstly, as compared to the 2-D prestall condition $(r / c=\infty)$, the separation point is postponed because of the effect of Coriolis force, which acts as a favorable pressure gradient tending to delay the separation to a point further downstream towards the trailing edge. It is shown that, as the radial location $r / c$ decreases, the separation point moves towards the trailing edge, and consequently the suction side distribution of the pressure coefficient moves towards lower levels (Chaviaropoulos \& Hansen, 2000), Fig. 8. The drop of the pressure coefficient along 
the suction side can be related to the separated area on the blades, which reduces in comparison with the 2-D $(r / c=\infty)$.

Secondly, in the stall incidence condition, leading edge separation bubbles are formed on the upper surface of the blade, at the root area, which delays the occurrence of massive separation. Beginning at the root of the blade, the bubble continues to stretch towards the trailing edge and at approximately $r / c=4$ where the bubble stretches all the way to the trailing edge, the flow separates over the whole airfoil (Dumitrescu \& Cardos, 2010) In the next section it is shown that there is a suction effect at the hub up to the mid-span which reduces the leading-edge bubble volume and produces a significant pressure drop along the suction side of the airfoils increasing, thus, the loading of the blade.

\section{Flow at high wind speeds}

At high wind speeds conditions ( TSR $\leq 3.0$ ), though the stall incidences are exceeded on the whole blade, the flow is partially separated on the inboard half-span of the blade and massively separated on the outboard half-span, Fig. 9.

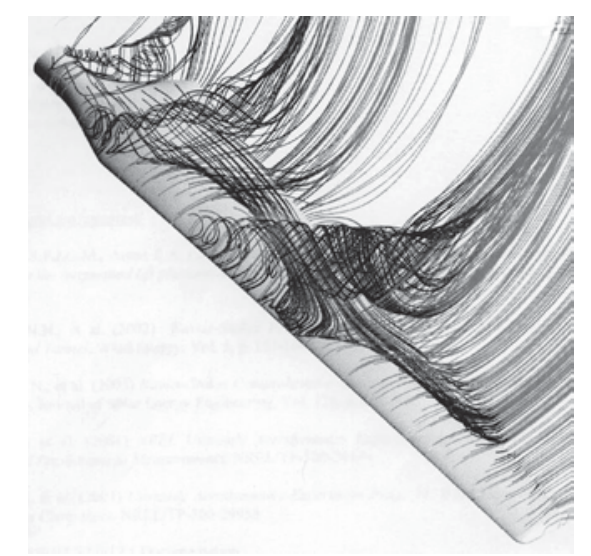

Fig. 9. Typical surface and 3-D streamlines on the blade suction side at high wind speeds $(\mathrm{TSR}=3.0)$.

This behavior, which is characterized by significantly increased lift coefficients as compared to the corresponding 2-D case, and by a delay of the occurrence of flow separation to higher angles of attack, is caused by the rotational augmentation at the root area of the blade. The knowledge extracted from experimental and computational visualizations of these rotational effects on the flow field along the blade can be used to develop a model for their prediction or the so-called stall-delay phenomenon.

The wind turbine blade sections, often operate with stall at low tip speed ratios and can undergo a delayed stall phenomenon on the inner part of span. As the wind velocity increases, the inboard regions of the blades are stalled, having $V_{w} / \Omega_{b} r>1$ for much of their operational time. The flow is neither two-dimensional nor steady, and is affected by rotation. As the root is approached the flow causes high values of tangential velocity and it behaves rather like the rotating flow over a stationary disk, Fig. 10 a), b). This is the result of 
a strong nonlinear interaction between the high wind speed and the rotational flow induced by a constant-speed rotor, which can be written as
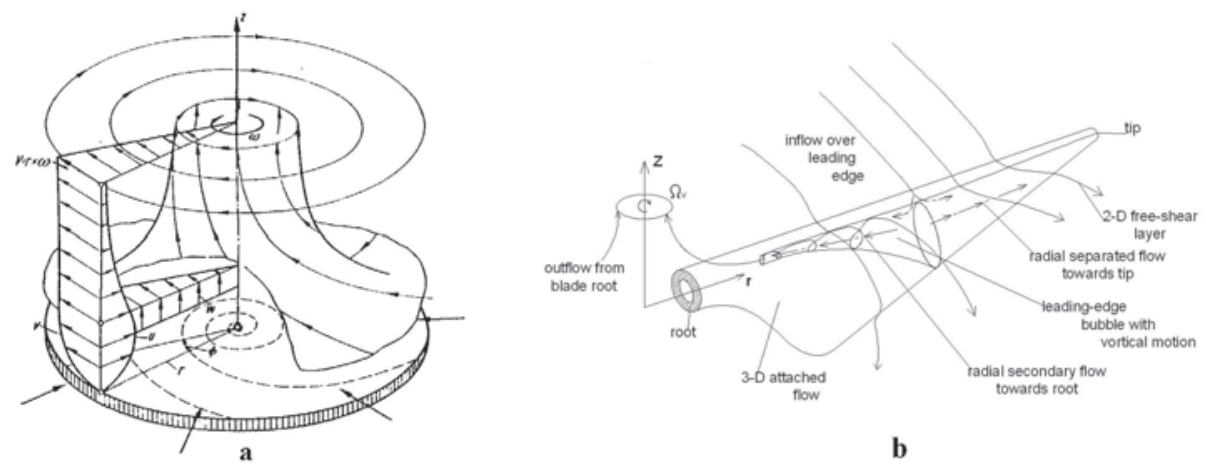

Fig. 10. Sucking-work mode of a wind turbine at high wind speeds (TSR $\leq 3.0)$ : a) concept of rotating flow over a stationary disk; b) model of the separated flow (Dumitrescu \& Cardoş, 2009)

$$
U_{r}=\Omega_{b}\left(\frac{V_{w}^{2}(1-a)^{2}}{\Omega_{b}^{2} r^{2}}+(1+d)^{2}\right)^{\frac{1}{2}} r=\Omega_{v}\left(\frac{r}{c}, \frac{V_{w}}{\Omega_{b} r}\right) r
$$

a form that suggest an outer rotational flow with angular velocity higher than that of the constant-speed rotor, $\Omega_{v}>\Omega_{b}$ and $\Omega_{v} / \Omega_{v}=-\Omega_{b} / \Omega_{b}$.

In contrast with the Glauert weak wake (Glauert, 1963), the strong potential vortex-like wake is responsible for all the 3-D rotational effects, affecting the boundary layer on the turbine blades once the phenomenon of stall is initiated. The enhanced rotational outer flow and the bottom boundary layer are the cause of the high-lift effect of the airfoils at high angle of attack, i.e. the stall delay.

\subsection{Boundary layer beneath a Rankine-like vortex}

The axisymmetric angular velocity (Ekman) boundary layer developing on the suction side of the rotor disk is used to illustrate the contribution of the suction effect of a strong wake on the chordwise surface pressure distribution and volume flux in the radial direction. Thus, consider an axisymmetric rotating flow of a viscous incompressible fluid over a disk of radius $R_{0}$ (Fig. 11).

The angular velocity of the disk $\Omega_{b}$ is constant and that of the fluid far away from the disk $\Omega_{v}$ may be an arbitrary function of the radius $r$, provided it is stable, i.e. $\frac{d\left(\Omega_{v} r^{2}\right)}{d r} \geq 0$ and near the axis the outer flow behaves locally like a rigid body rotation, i.e. $r \rightarrow 0: \frac{d \Omega_{v}}{d r} \rightarrow 0, \Omega_{v} \rightarrow \Omega_{b}$. The Rankine-like vortex assumed for the outer rotational flow satisfies both conditions and can be described by the vortex circulation $\Gamma_{v} \equiv \Omega_{v} r^{2}$ given by the empirical formula (Vatistas et al., 1991) 


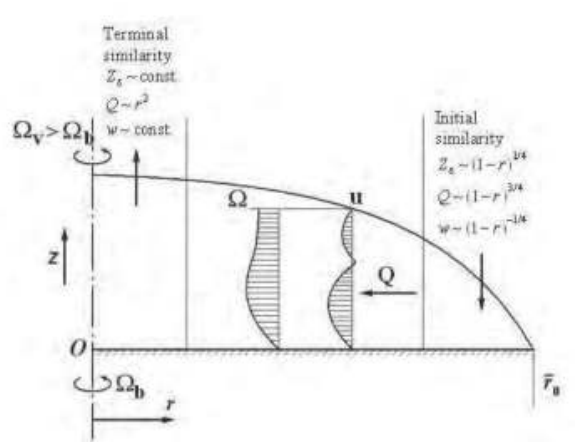

Fig. 11. Boundary layer on a rotating disk slower than the outer flow.

$$
\Gamma_{v}=\Omega_{b} R_{0}^{2} \sqrt{\frac{1+\frac{r_{h}^{4}}{R_{0}^{4}}}{1+\left(\frac{r}{r_{h}}\right)^{4}}}\left(\frac{r}{r_{h}}\right)^{2},
$$

where $r_{h}$ is the radius of forced vortex core.

This vortex model avoids the discontinuity of the velocity derivatives at the point of transition from free to forced modes and gives the possibility of the description of the flow near the hub.

For the inertial system of cylindrical coordinates $(r, \theta, z)$ considered in Fig. 10, the laminar boundary layer equations write as (Rott \& Lewellen, 1966)

$$
\begin{gathered}
\frac{\partial(r u)}{\partial r}+\frac{\partial(r w)}{\partial z}=0 \\
\frac{\partial\left(u^{2}\right)}{\partial r}+\frac{u^{2}-v^{2}}{r}+\frac{\partial(u w)}{\partial z}=-\frac{1}{\rho} \frac{\partial p}{\partial r}+\mathrm{v} \frac{\partial^{2} u}{\partial z^{2}}, \\
\frac{\partial(u v)}{\partial r}+\frac{2 u v}{r}+\frac{\partial(v w)}{\partial z}=\mathrm{v} \frac{\partial^{2} v}{\partial z^{2}}
\end{gathered}
$$

with $\frac{\partial p}{\partial z}=0$, where $(u, v, w)$ are the velocity components, $\mathrm{p}$ is the static pressure, $\rho$ is the fluid density and $v=\frac{\mu}{\rho}$ is the kinematic viscosity.

The boundary conditions at the surface of the disk and the edge of boundary layer are

$$
\begin{aligned}
& z=0: \quad u=w=0, v=\Omega_{b} r=\frac{\Gamma_{b}}{r}, \\
& z=Z_{\delta}: \quad u=0, v=V=\Omega_{v} r=\frac{\Gamma_{v}}{r} .
\end{aligned}
$$


The outer flow radial momentum equation becomes then $\frac{V^{2}}{r}=\frac{1}{\rho} \frac{d p}{d r}$ and the pressure in the outer flow is

$$
C_{p s}=\frac{p-p_{0}}{p_{0}-p_{c}}=\frac{\int_{0}^{r} \frac{\Gamma_{v}^{2}}{r^{3}} d r}{\int_{0}^{1} \frac{\Gamma_{v}^{2}}{r^{3}} d r}-1
$$

where $C_{p s}$ is the suction pressure coefficient normalized by the pressure at the vortex center, and the subscripts $c$ and 0 indicate properties at the vortex center and the edge of disk, respectively.

The tangential and radial momentum-integral equations may be derived from equations (26) and (27) for radial and angular velocity profiles which satisfy the boundary conditions on the disk surface and the smoothness requirements at the edge of the boundary layer

$$
\begin{aligned}
& u=U(r) f^{\prime}\left(\frac{z}{Z_{\delta}}\right), f^{\prime}(0)=0, f^{\prime}(1)=0, f^{\prime \prime}(1)=0 \\
& \frac{v-\Omega_{b} r}{V-\Omega_{b} r}=g\left(\frac{z}{Z_{\delta}}\right), g(0)=0, g(1)=1, g^{\prime}(1)=0
\end{aligned}
$$

Outside the boundary layer, the tangential velocity $V(r)=\frac{\Gamma_{v}}{r}$ is assumed to be some given function, Eq. (24), while the radial velocity vanishes. The variables are the boundary-layer thickness $Z_{\delta}$ and the radial volume flux $Q=\int_{0}^{Z_{\delta}} r u \mathrm{~d} z$.The momentum-integral equations under these conditions are

$$
\begin{gathered}
\frac{d}{d r}\left[Q\left(\Gamma_{f}-\Gamma_{b}\right)\right]-\lambda_{1} Q \frac{d \Gamma_{f}}{d r}=\lambda_{1} \frac{r^{2} \tau_{\theta}}{\rho}, \\
\lambda^{2} \frac{d}{d r}\left[\frac{Q^{2}}{r Z_{\delta}}\right]+\frac{Z_{\delta}}{r^{2}}\left[\lambda_{3}\left(\Gamma_{f}-\Gamma_{b}\right)^{2}+\lambda_{4} \Gamma_{b}\left(\Gamma_{f}-\Gamma_{b}\right)\right]=-\frac{r \tau_{r}}{\rho},
\end{gathered}
$$

where $\lambda_{1}, \lambda_{2}, \lambda_{3}$ and $\lambda_{4}$ are profile form parameters defined in Table 1 (Rott \& Lewellen, 1966)

\begin{tabular}{|l|c|c|c|c|c|c|c|c|}
\hline Flow & $f^{\prime}(\eta)$ & $g(\eta)$ & $\lambda_{1}$ & $\lambda_{2}$ & $\lambda_{3}$ & $\lambda_{4}$ & $C_{1}$ & $C_{2}$ \\
\hline Laminar & $6.75 \eta(1-\eta)^{2}$ & $\eta(2-\eta)$ & 2.5 & 1.372 & 0.467 & 0.667 & 2 & 12 \\
\hline Turbulent & $1.69 \eta^{1 / 7}(1-\eta)^{2}$ & $\eta^{1 / 7}$ & 4.93 & 1.63 & 0.222 & 0.250 & 0.0225 & 0.0513 \\
\hline
\end{tabular}

Table 1. Various parameters used in the axisymmetric boundary layer. 
The Blasius shear laws for three-dimensional flow (Schlichting, 1979) cover both the laminar and turbulent case

$$
\begin{gathered}
|\tau|=C \rho V_{\infty}^{2}\left(1+\frac{U_{m}^{2}}{V_{\infty}^{2}}\right)^{1-\frac{\mu}{2}}\left(\frac{v}{V_{\infty} z_{\delta}}\right)^{\mu}, \\
\tau_{\theta}=C_{1} \rho V_{\infty}^{2}\left(1+\frac{U_{m}^{2}}{V_{\infty}^{2}}\right)^{\frac{1-\mu}{2}}\left(\frac{v}{V_{\infty} z_{\delta}}\right)^{\mu}, \tau_{r}=C_{2} \rho V_{\infty} U_{m}\left(1+\frac{U_{m}^{2}}{V_{\infty}^{2}}\right)^{\frac{1-\mu}{2}}\left(\frac{v}{V_{\infty} z_{\delta}}\right)^{\mu},
\end{gathered}
$$

where $U_{m}$ is equal to the maximum value of $u$ and following Blasius, $\mu=1$ (laminar flow), $\mu=1 / 4$ and $C=0.0225$ (turbulent flow).

This is the final result of the analytical formulation and the equations (32) and (33) must be integrated numerically for a particular circulation function $\Gamma_{v}$ (Eq. (24)) with an appropriate choice of the boundary layer form parameters.

The onset of vortical flows like tornadoes can be visible on the constant-rotational speed rotor at low speed ratios $(\lambda<3.0)$. At the root area of the blade these flows generally behave like a vacuum pump which is featured by volume flow rate $(Q)$ and suction pressure $\left(C_{p s}\right)$.

Figures 12 and 13 show such characteristics induced by the wake modeled as a Rankine-like vortex with its axis normal to the rotor disk for different tip speed ratios. The suction characteristics show that the effects of the disk defined by $V_{w} / \Omega_{b} r \geq 1$ decrease rapidly with the radius towards the edge of the disk $\left(V_{w} / \Omega_{b} r=1\right)$ and have maximum values near the hub (here $r_{h}=0.2 R$ ).

On the other hand, large radial volume fluxes close to the hub along with the previous results (Fig. 7a) indicate a 3-D attached boundary layer with small leading-edge separation bubbles at high angles of attack.

Therefore, at low tip speed ratios and close to the hub, there is essentially a 3-D attached flow field and a theoretical description in this region can be performed by viscous-inviscid interactive flow models.

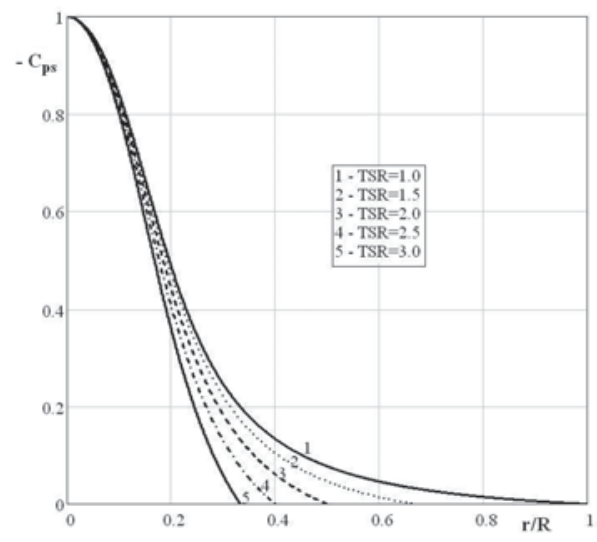

Fig. 12. Radial suction pressure induced by the wake at the $r_{h} / R=0.2$. 


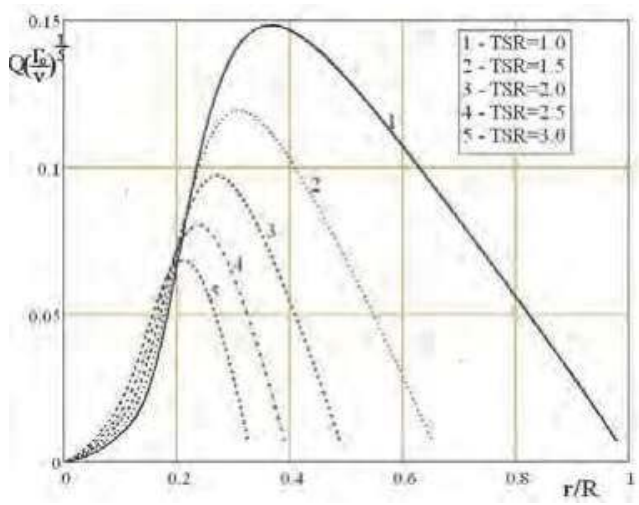

Fig. 13. Radial volume flow rate in the boundary layer beneath the wake at $r_{h} / R=0.2$.

\subsection{Blade-wake interaction}

Flow at low tip speed ratios is dominated by a vortex-like wake which triggers the stall delay phenomenon on the inboard half-rotor disk. The dual working mode of the rotor blades as a stationary disk in a fast rotating flow near the hub-sucking mode, and as the rotating disk in the outboard separated flow-pumping mode, are clearly visible from their pressure distributions when the wind turbine operates at $\lambda \leq 3.0$.

On the upper side of the inner blade sections there are two suction pressure fields: one is azimuthally uniform generated by circulation augmentation of wake and the other is the aerodynamic non uniform pressure field with a suction spike at the leading-edge (Fig. 4). These two pressure fields interact and give rise to the radial effects which contribute to stall delay, as well as to a higher lift coefficient at the root area of the blade. There are two main effects: superimposed pressure fields of wake (Fig. 12) and of blade (Fig. 4), which decrease the suction spikes at the leading edge until they cancel, and a radial flow effect induced by the volume flow merger in the boundary layers of wake and blade, resulting in velocity profile skewing and boundary-layer energizing (no separation). Assuming the conservation of circulation once the phenomenon of stall is initiated (here at $\lambda=3.0$ ) the wake-blade pressure interaction for the increased wind speed $(\lambda<3.0)$ is a pressure redistribution process of pressures $\left(C_{p U}, C_{p s}\right)$ along the chord at constant circulation (lift). The rule of pressure redistribution is sketched as is shown in Fig. 14.

As we will show in the next section, the phenomenon of stall-delay can be described as a three step process: generation of the strong wake by the clustering of shed vortices from the inboard blade segments at $\lambda=3.0$, chordwise pressure redistribution at the constant value of circulation at the start regime $(\lambda=3.0)$, for $\lambda<3.0$, followed by circulation decay for increasing radius. The pressure redistribution process is companied by the move of the pressure center to the midchord.

\subsection{Application}

The sucking suction effect is affected by two key non-dimensional parameters $\left(\frac{V_{W}}{\Omega_{b} r}, \frac{r}{c}\right)$. Figures 15a and 15b illustrate the influence of these parameters on the chordwise pressure 


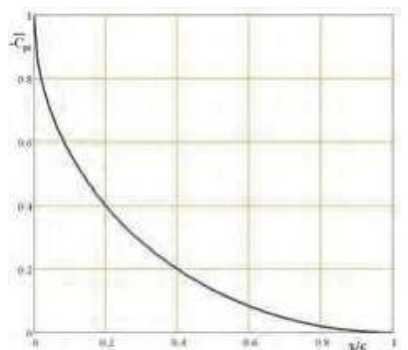

inviscid load (no sucking)

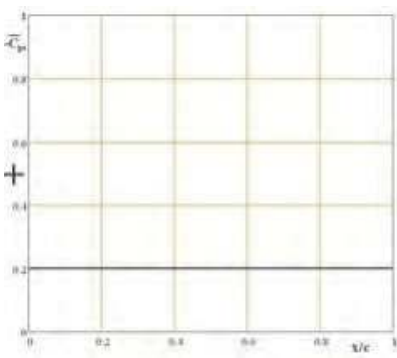

sucking load

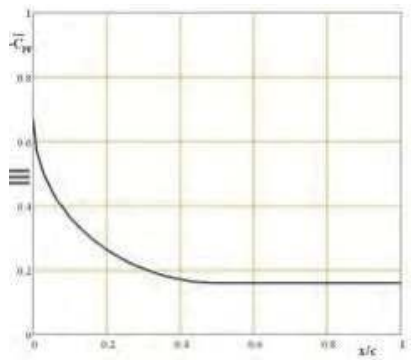

redistributed load $\left(C_{L}=\right.$ const $)$

Fig. 14. The rule of the suction pressure with superimposed rotation effect in a normalized plan: $\bar{C}_{p}=C_{p} /\left|C_{p m}\right|$

distribution for the previous hypothetic blade, with constant post-stall incidence and chord along the span $\left(\left|C_{p m}\right|=10\right)$. The figures also indicate the boundary layer state on the upper surface: $S$-separation and $R$ - reattachment.
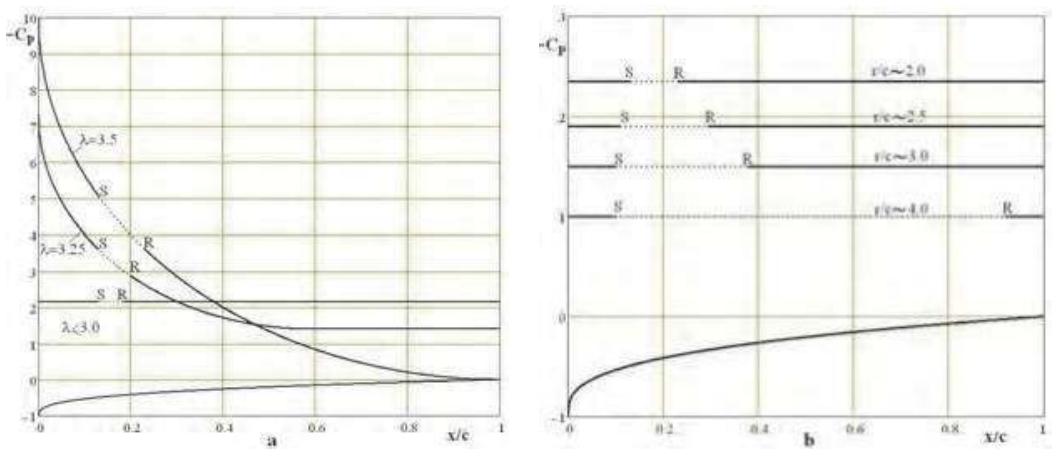

Fig. 15. Influence of TSR and $r / c$ ratio on the suction pressure: a) $C_{p}(T S R)$ for $r / c=2$; ) $C_{p}(r / c)$ for $T S R \leq 3$.

\section{Model for delayed stall regime}

Stall occurs at an airfoil when flow separates at high angles of attack, typically $>15^{0}$, beyond this angle the 2-D lift coefficient drops significantly. It has long been known (Himmelskamp, 1947; Ronsten, 1992; Snel et al., 1993), however, that the observed power curve under conditions where most parts of the blade are stalled is consistent with significantly higher lift coefficients. The lack of a conceptual model for the complex 3-D flow field on the rotor blade, where stall begins, and how it progresses, has hindered the finding of an unanimously accepted solution. This section aims at giving a better understanding of the delayed stall events.

To interest an engineer in stall-delay one might cite the fact that the inboard regions of the blades are stalled for much of their operational time. Thus predicting blade loads and the power output during stall is very important in making good predictions of wind turbine 
performance. With a physicist one could reason that rotational stall admits interesting transient states from 3-D inboard delayed stall in partially separated flow to 2-D outboard massively separated flow. Here, the detailed physical understanding of the viscous flow surrounding the hub area is dominated by the interaction between the vorticity and pressure fields and their dynamics. However, all the existent models have been developed more or less intuitively without a basic fluid dynamics support. Six different models mostly used to correct the airfoil characteristics for stall delay, including a wide range of different assumptions, were recently analyzed (Breton et al., 2008). The conclusion was that none of the six models studied correctly represented the flow physics, and that this was ultimately responsible for their lack of generality.

\subsection{New physics-based model}

Flow Topology of Delayed Stall. There are two main flow regimes pertinent to wind turbine rotors: one is a flow close to rotating disk in axial flow at low velocity, practically at rest, $(\lambda>3$, Fig. 2), and another is a rotating flow over a stationary or slowly rotating disk (Fig. 9). The last can produce delayed stall on the wind turbine blade sections operating at low tip speed ratios $(\lambda \leq 3)$.

The key to the stall-delay phenomenon is the rotational flow surrounding the root area of the blade, i.e. the wake rotation. At low tip speed ratios (or high wind speeds), just as a vortex is shed from each tip blade, a vortex is also shed beginning from the section $\left\{\frac{V_{w}}{\Omega_{b} r}=1\right\}$ of each blade. Then the blade vortices from $\left\{\frac{V_{w}}{\Omega_{b} r} \geq 1\right\}$ span will each be a line vortex running axially to the center of the rotor. The direction of rotation of all $\left\{\frac{V_{w}}{\Omega_{b} r} \geq 1\right\}$ span vortices will be the same, forming a core (or root) vortex of total strength $\Gamma_{v} \gg \Gamma_{b}=\Omega_{b} r^{2}$. The root vortex is primarily responsible for inducing the rotational 3-D effects on blades. The acquisition of the tangential component of air velocity is compensated for by a fall in the static pressure (suction) in the wake.

There are two common characteristics which most strong potential vortices exhibit, like those produced at the blade root; the first is that the vortex velocity field above the surface boundary layer is always dominated by the tangential velocity component, while the second shows that the balance of forces between the flow outside the boundary layer and vortex core is governed by a balance between the centrifugal force and radial pressure gradient $\left(\frac{1}{\rho} \frac{\partial p}{\partial r}=\frac{V^{2}}{r}\right)$. In this working mode the centrifugal force has a strong stabilizing effect on the boundary layer through the favorable radial pressure gradient which occurs. In contrast with the pumping - working mode at high tip speed ratios, now the centrifugal forces produce a centerwise suction effect, resulting mainly in boundary layer stabilizing against the separation. On the other hand, in the presence of a solid surface (blade), this balance of forces is disrupted by the friction retardation of the tangential flow close to the boundary. Under such a condition, the radial pressure gradient proceeds to drive the retarded boundary flow along the surface towards the center, resulting in large radial velocities residing close to the surface. Above the boundary layer where the cyclostrophic balance is still intact, the radial velocity inevitably falls to zero. This gives rise to an inflexion point in 
the radial velocity profile. At some location close to the axis, this inward flowing air moves away from the solid boundary and effuses into a vortex core. The radial pressure gradient has a very strong stabilizing effect on the boundary layer and acts to revert it to its reattaching, and even more to its laminar state upstream of the effusing core.

Modeling of Stall-Delay. The acquisition of the enhanced rotational flow (tangential component of velocity) by the increase in the kinetic energy of wind, associated with a strong suction of the air at the hub is governed by conservation of angular momentum, like the potential vortex, and conservation of energy:

$$
\begin{gathered}
\Gamma_{v}=\text { const. } \\
\frac{p}{\rho}+\frac{\Gamma_{v}}{2 r^{2}}=\text { const. }
\end{gathered}
$$

Then, as the blade section moves through the air a circulation $\Gamma_{\text {airfoil }}$ develops around it. In order to comply with Kelvin's theorem,

$$
\frac{D \Gamma}{D t}=\frac{\Gamma_{\text {airfoil }}+\Gamma_{\text {wake }}}{\Delta t}=0,
$$

a starting vortex $\Gamma_{\text {wake }}$ must exist such that the total circulation around a line that surrounds both the airfoil and the wake remains unchanged. Since the circulation of the sectional airfoil, where the phenomenon of stall was initiated at $\lambda=3.0$, is practically the blade circulation due to rotation, $\Gamma_{\text {airfoil }}=\Gamma_{b}=V_{w}^{2} / \Omega_{b}=\Omega_{b} R^{2} / \lambda^{2}$ (at $r / R=\lambda^{-1}$ the sectional airfoil is stalled for $\lambda=3.0)$, then $\Gamma_{\text {wake, } 0}=-\Gamma_{b}(r=R / 3.0)$.

The wake's circulation, which is constant up to the hub induces a suction pressure along the upper side of blade (Fig. 16) stabilizing the boundary layer against the separation at poststall angles of attack (angles of 30 degrees exist currently at the blade root), followed by forming of a leading-edge separation bubble. The bubble at the innermost sectional airfoil has almost no effect on integrated loads, because it is never more than a few percent of the chord in length, and the airfoil acts as in an ideal fluid flow.

The phenomenon of stall-delay can be described as a three step process: rise of strong wake by clustering of vortices shed from the inboard blade span at $\lambda=3.0$, inviscid (pressure) load redistribution along the airfoil chord at constant circulation or $C_{L, I N V}$ of the start regime $(\lambda=3.0)$ for increased suction pressure at $\lambda<3.0$, followed by spanwise circulation or lift decay involving the stretching of separation bubble surface all the way to the trailing edge for increasing radius; beyond that blade section $\left(r / R=\lambda^{-1}\right)$ the flow is separated over the whole airfoil and the leading-edge stall occurs.

The specific mechanism for circulation (or lift) decay is not presently known. However, a measure of the degree of radial instability of the boundary layer and implicitly the lift decay can be obtained from the sucked volume flow rate (Fig. 13) into the boundary layer beneath the Rankine vortex-like wake. Thus, the maximum sucked flow rate would correspond to the minimum volume of the separation bubble and $C_{L, I N V}$. Consequently $C_{L}$ decay can be supposed to follow the flow rate decrease, $\frac{d C_{L}}{d r} \sim \frac{d Q}{d r}$, where the zero flow rate indicates the full stall occurring. More accurately, the curves of the volume flow rate show the state of 


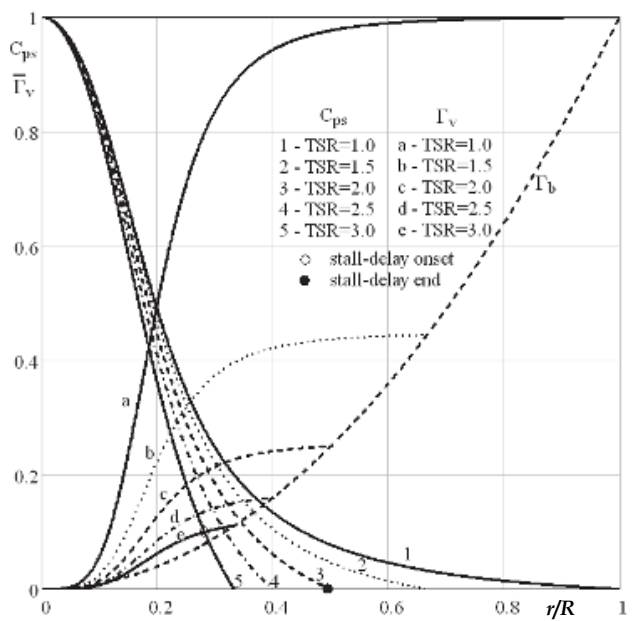

Fig. 16. Circulation $\Gamma / \Omega_{b} R^{2}$ and pressure $\left(C_{p s}\right)$ correlation for $T S R \leq 3.0$.

boundary layer, namely larger values of the flow rate signify smaller bubble volumes and thinner boundary layers for reducing tip speed ratios. Further, it can be easily found that a thicker boundary layer with distributed vorticity $(\lambda=3.0)$ is more stable, while a thinner boundary layer with more concentrated vorticity $(\lambda \rightarrow 1.0)$ is less stable. Since the lift decay law depending on the stability of the boundary layer, is different from that suggested by Fig. 13, tending to be slower for higher $\lambda$ and steeper for lower $\lambda$, an average decay corresponding to $\lambda=2.0$ is assumed. Therefore, the decay is assumed to be terminated for all regimes at midspan, i.e. the end of the flow regime for $\lambda=2.0$. This conclusion is consistent with the result found in (Dumitrescu \& Cardoş, 2010), which indicates the full separation at $r / c=4$.

\begin{tabular}{|c|c|c|}
\hline Point & Flow Structure & Forces \\
\hline 1 & $\begin{array}{c}\text { The attached 3-D boundary layer with } \\
\text { leading-edge separation bubble }\end{array}$ & $\begin{array}{c}\text { Exceed 2-D } C_{L \text { max }} \text { extrapolate linear } \\
\text { regime }\end{array}$ \\
\hline 2 & $\begin{array}{c}\text { The separation bubble is sucked and its } \\
\text { volume reduces }\end{array}$ & $\begin{array}{c}\text { Maximum lift, } C_{L, I N V} \text { at } \lambda=3.0, \text { followed } \\
\text { by suction pressure redistribution and } \\
\text { movement of pressure center to } \\
\text { midchord; no correction to drag }\end{array}$ \\
\hline 3 & $\begin{array}{c}\text { Thick skewed 3-D boundary layer with } \\
\text { stretching bubble }\end{array}$ & $\begin{array}{c}\text { Gradual decay of lift for } r>r_{0} ; \text { no } \\
\text { correction to drag }\end{array}$ \\
\hline 4 & $\begin{array}{c}\text { The bubble breaks away from the } \\
\text { trailing edge and forms a free shear } \\
\text { layer }\end{array}$ & Readjust to stall regime \\
\hline
\end{tabular}

Table 2. Delayed stall events on a wind turbine blade. 

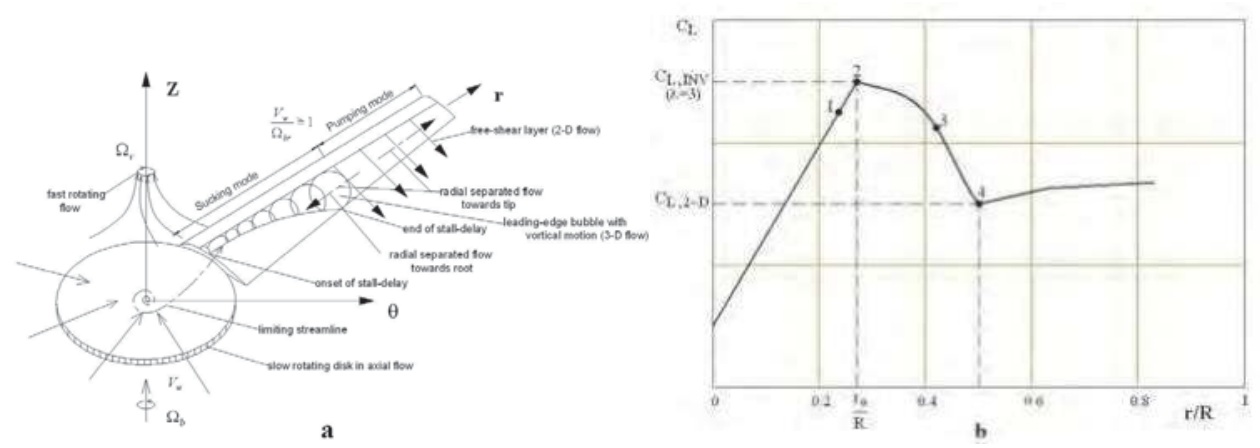

Fig. 17. Schematic showing the essential 3-D flow topology a) and delayed stall events b) on a wind turbine blade

The various stages of the delayed stall process are summarized schematically in Fig. 17. and Table 2. Stage 1 represents the delay in the onset of separation in response to reduction in adverse pressure gradients produced by the influence of the strong vortex wake at the root. The phenomenon is initiated at $\lambda=3.0$, where the suction pressure equalizes to the kinetic energy of wake (Fig. 16) resulting mainly in boundary layer reattaching and the rise of a leading edge separation bubble, a stable focus as topological entity.

Stage 2 of the delayed stall process involves the suction pressure redistribution of the start pressure distribution with the conservation condition of its integrated load and the simultaneous move of pressure center to the midchord; as the suction/tip speed ratio increases/decreases the volume of the leading-edge separation bubble is reduced. The start pressure distribution is known from the inviscid aerodynamic loading solution over the chord; the pressure redistribution continues up to no suction peak exists at the leading-edge and even more no suction surface pressure gradient exists. On the other hand, the pressure redistribution is associated with a vorticity concentration process, which influences the stability of boundary layer.

Stage 3 is the sectional circulation (lift) decay which occurs at greater radii than the start location, where the separation bubble surface stretches all the way to the trailing edge. Beyond that zero flow rate location the flow is separated over the whole airfoil so that full stall occurs. By reason of the boundary layer stability it is inferred that the stall-delay effects are present up to a $50 \%$ of span for all the regimes.

Stage 4 is the relaxation to stall regime, which begins just as the integrated normal load of 2$\mathrm{D}$ stall, $\mathrm{C}_{\mathrm{NS}}$, is reached (end of delayed stall) and continues as far as the recovery of the specific pressure distribution (suction peak at the leading edge) is achieved.

\subsection{Application}

The combined experimental rotor (Schepers et al. 1997) of the Renewable Energy Laboratory (NREL) is chosen to illustrate the stall-delay model proposed for a wind turbine blade. The rotor uses the NREL S 809 airfoil and a simpler BEM method. In this example, the one parameter pressure distribution on the upper surface of blade at the root with $\left|C_{p m}\right|=11(\alpha=22 \mathrm{deg})$ is imposed and the hub is at approximately $0.20 \mathrm{R}$. The curves plotted in Fig. 19 are calculated with these conditions: the onset of stall-delay at $\lambda=3.0, r_{0} / R=0.268$, $C_{L, I N V(\lambda=3)}=2.35$ and a linear approximate distribution of normal load decay (Fig. 18). 

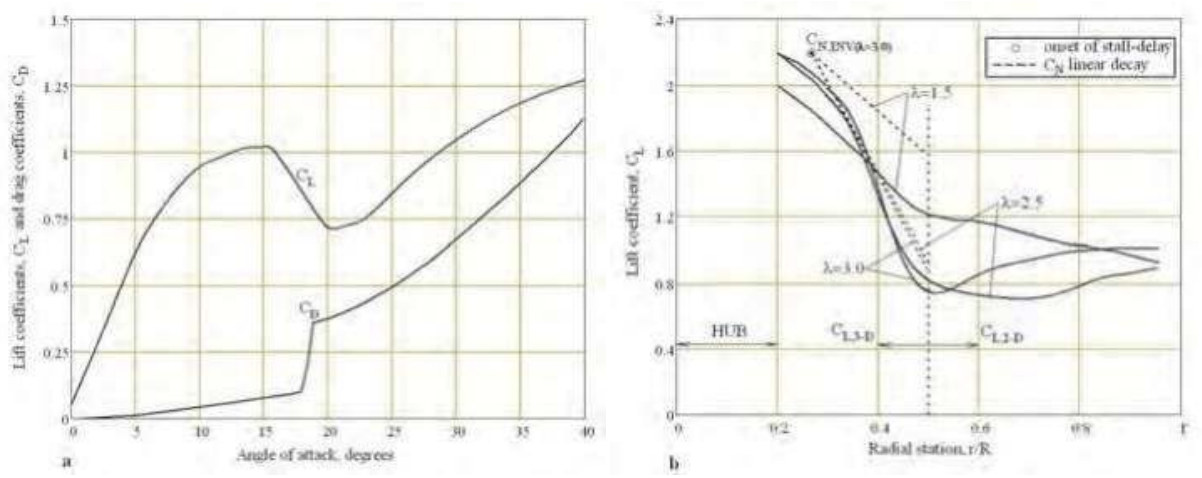

Fig. 18. Sectional aerodynamic and stall-delay characteristics used for the application: a) $C_{L}, C_{D}$ coefficients for $S 809$ airfoil at $\left.\operatorname{Re}=10^{6} ; \mathrm{b}\right) C_{L, 3-D}$ model.
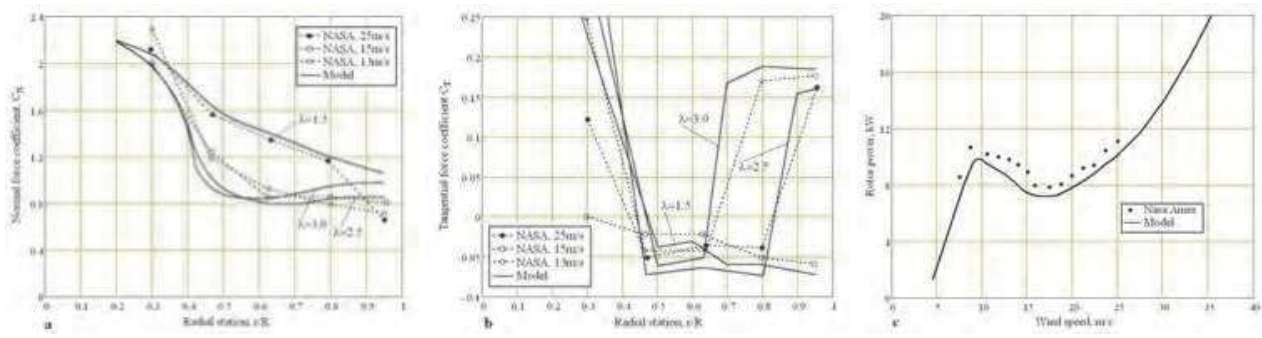

Fig. 19. Predicted performance comparison and NASA-Ames data: a) normal force coefficients; b) tangential force coefficients; c) rotor power.

The comparison of predicted and available measured $C_{N}, C_{T}$ and $P$ are shown in Fig. $19(\mathrm{a}, \mathrm{b}$, c), for wind speeds $13 \mathrm{~m} / \mathrm{s}, 15 \mathrm{~m} / \mathrm{s}$ and $25 \mathrm{~m} / \mathrm{s}$. Excepting predicted $C_{T}$ at the blade root, the agreement between predictions and measurements is reasonably good. Most likely, at the root area of the blade there are computational and experimental uncertainness, and better more exist uncertainness for predictions. However, the present stall-delay model is the most comprehensive one able to capture much of the actual physics of flow.

\section{Chapter review}

The phenomenon of stall-delay has been shown to be an important consideration in wind turbine design because, in the presence of turbulence, it ultimately generates the loads with the highest peak-to-peak fatigue cycles for both blade and rotor shaft bending (Tangler, 2004). It has been shown that stall-delay is characterized by a favorable delay in onset of flow separation to higher angles of attack for the inboard regions of the blades. This is followed by the less favorable event of leading-edge separation bubble stretching. As long as the bubble surface stays over airfoil, it acts to decay the lift gradually. Then, the leadingedge separation occurs. The stall-delay begins at the hub and crosses varied partially separated flow regions over the inboard half-rotor disk, depending on the wind speed. Therefore, the consideration of stall-delay phenomenon represents a necessary refinement in 
the rotor design process which will more accurately define the performance and power control at high wind speeds.

While the prediction of the conditions for stall-delay onset and their subsequent effects clearly forms an essential part of any rotor design process, it has been shown that this is a problem not yet fully understood, nor easily predicted. For engineering analyses, the modeling of stall-delay still remains a particularly challenging problem. This is mainly because of the need to balance physical accuracy with computational efficiency and/or the need to formulate a model of stall-delay in a particular mathematical form. To this end, a number of semi-empirical models have been developed for use in wind turbine design work. A discussion of these semi-empirical methods has been recently presented, along with the conclusion that none of the six most known models correctly represented the physics of flow (Breton et al., 2008). Generally, predictions are good when measurements are available for validation or empirical refinement of the model, but their capabilities for general blade parameters (twist, taper and airfoil shape) are less certain.

A conceptualization of the complex 3-D flow field on a rotor blade, where stall begins, and how it progress, has been proposed, along with a reasonably simple model that complements the 2-D airfoil characteristics used to predict rotor performance. This concept considers that the 3-D flow field near the hub behaves like the rotational flow over a stationary disk, inducing a strong suction pressure towards the center. Here, the centrifugal force has a strong stabilizing effect on the boundary layer, in which the rotor is acting as a vacuum pump on the separated volume of bubble. The rough model has shown encouraging results, but some uncertainties remain in the prediction of the sectional circulation decay and also in the proper validation of predictions with more measured and CFD computed airloads on the rotor. The more correct physical description provided by this present model should give more coherence and consistence of future experimental and computational studies on the stall-delay phenomenon.

\section{References}

Banks, W. \& Gadd, G. (1963). Delayed effect of rotation on laminar separation, AIAA Journal, Vol. 1, No. 4.

Bjorck, A. (1995). Dynamic stall and three-dimensional effects, FFA TN-1995-31.

Breton, S.Ph.; Coton, F.N. \& Moe, G. (2008). A study of rotational effects and different stall delay models using a prescribed wake vortex scheme and NREL phase VI experiment data, Wind Energy, Vol. 11, pp. 459-482.

Burton, T.; Sharpe, D.; Jenkins, N. \& Bossanyi, E. (2001). Wind Energy Handbook, Wiley, Chichester.

Cebeci, T. \& Cousteix, J. (1999). Modeling and computation of boundary layer flows, SpringerVerlag, Berlin.

Chaviaropoulos, P.K. \& Hansen, M.O.L. (2000). Investigating three-dimensional and rotational effects on wind turbines blades by means of quasi-3-D Navier-Stokes solver, Journal of Fluids Engineering, Vol. 122, pp. 330-336.

Corrigan, J.J. \& Schlichting, J.J. (1994). Empirical model for stall delay due to rotation, AHS Aeromechanics Specialist Conference, San Francisco, CA, 8.4-(1-15).

Corten, G.P. (2001). Flow separation on wind turbine blades, PhD Thesis, University of Utrecht. 
Drela, M. (1989). XFOIL: An analysis and design systems for low Reynolds number airfoils, Conference on low Reynolds number airfoil aerodynamics, University of Notre Dame, June 1989.

Du, Z. \& Schling, M.S. (1998). A 3-D stall-delay model for horizontal axis wind turbines performance prediction, AIAA-98-0021.

Du, Z. \& Selling, M.S. (2000). The effect of rotation on the boundary layer of a wind turbine blade, Renewable Energy, Vol. 20, pp. 167-181.

Duque, E.P.N.; Burkland, M.D. \& Johnson, W. (2003). Navier-Stokes and comprehensive analysis performance predictions of the NREL phase VI experiment, Journal of Solar Energy Engineering, Vol. 125, No. 4, pp. 457-467.

Dumitrescu, H. \& Cardoş, V. (2004). Rotational effects on the boundary layer flow in wind turbines, AIAA Journal, Vol. 42, No. 2, pp. 408-411.

Dumitrescu, H.; Cardoş, V. \& Dumitrache, A. (2007). Modelling of inboard stall delay due to rotation, The Science of Making Torque from Wind, Journal of Physics: Conference series, Vol. 75, IOP Publication doi: 10.1088/1742-6569/1/012022.

Dumitrescu, H. \& Cardoş, V. (2009). Inboard boundary layer state on wind turbine blades, ZAMM, Vol. 89, No. 3, pp. 163-173.

Dumitrescu, H. \& Cardoş, V. (2010). Analysis of leading-edge separation bubbles on rotating blades, J. Aircraft, Vol. 47, No. 5, pp. 1815-1819.

Fletcher, T.M.; Brown, R.E.; Kim, D.H. \& Kvon, O.J. (2009). Predicting wind turbine blade loads using vorticity transport and RANS methodologies, European Wind Energy Conference and Exhibition, Marseille, March.

Fogarty, L.E. \& Sears, W.R. (1950). Potential flow around a rotating, advancing cylindrical blade, Journal of Aeronautical Sciences (Reader Forum), Vol. 17 No. 9, pp. 599.

Fogarty, L.E. (1951). The laminar boundary layer on a rotating blade, Journal of Aeronautical Sciences, Vol. 18, No. 4, pp. 247-252.

Glauert H. (1963). Windmills and fans, in Aerodynamic Theory, Vol. 14, edited by Durand W.F., Dover ed.

Hansen, A.C. \& Butterfield, C.P. (1993). Aerodynamics of horizontal-axis wind turbines, Annual Review of Fluid Mechanics, Vol. 25, pp. 115-149.

Head, R.M. (1958). Entrainment in turbulent boundary layers, British Aeronautical Research Council, Report 3152, Sept. 1958.

Himmelskamp, H. (1947). Profile investigations on a rotating airscrew, (PhD dissertation, Götingen, 1945), MAP Volkenrode, Report and Translation No. 832.

Lakshminarayana, B. \& Govindan, T.R. (1981). Analysis of turbulent boundary layer on cascade and rotor blades of turbomachinery, AIAA Journal Vol. 19, No. 10, pp. 13331341.

Lock, R.C. \& Williams, B.R. (1987). Viscous-inviscid interactions in external aerodynamics, Progress in Aerospace Sciences, Vol. 24, No. 2, pp. 51-171.

Ludwieg, H. \& Tillman, W. (1949). Investigation of the wall shearing stress in turbulent boundary layers, NACA TM 1285.

Mager, A. (1951). Generalization of boundary layer momentum-integral equations to threedimensional flows including these of rotating systems, NACA Report 1067.

McCroskey, W.J. \& Dwyer, H.A. (1969). Methods of analyzing propeller and rotor boundary layers with crossflow. NASA SP-228, pp. 473-514. 
Narramore, J.C. \& Vermeland, R. (1992). Navier-Stokes calculations of inboard stall delay due to rotation, Journal of Aircraft, Vol. 29, No. 1.

Ronsten, G. (1992). Static pressure measurements on a rotating and non-rotating $2.375 \mathrm{~m}$ wind turbine blade. Comparison with 2-D calculations, Journal of Wind Engineering and Industrial Aerodynamics, Vol. 39, No. 1-3, pp. 105-118.

Rott, N. \& Lewellen, W.S. (1966). Boundary layers and their interactions in rotating flows, in Progress in Aeronautical Sciences, Vol. 7, pp. 111-144.

Schepers, J.G.; Brand, A.J.; Bruining, A.; Graham, J.M.R.; Hand, M.M.; Infield, D.G.; Paynter, R.J.H. \& Simms, D.A. (1997). Final report of IEA Annex XIV: field rotor aerodynamics, Technical Report ECN-C-97-027.

Schlichting, H. (1979). Boundary layer theory, McGraw-Hill, New York.

Sears, W.R. (1950). Potential flow around a rotating cylindrical blade, Journal of Aeronautical Sciences (Reader Forum), Vol. 17 No. 9, pp. 183-184.

Shen, W.Z. \& Sørensen, J.N. (1999). Quasi-3-D Navier-Stokes model for a rotating airfoil, Journal of Computational Physics, Vol. 150, pp. 518-548.

Snel, H.; Houwink, R.; van Bussel, G.J.W. \& Bruining, A. (1993). Sectional prediction of 3-D effects for stalled flow on rotating blades and comparison with measurements, 1993 European Community Wind Energy Conference Proceedings, Lübeck-Travemünde, Germany, pp. 395-399.

Sørensen, J.N. (1986). Prediction of the three-dimensional stall on wind turbine blade using three-level, viscous-inviscid interaction model, Proc. of EWEC, pp. 429-435.

Sørensen, N.N.; Michelsen, J.A. \& Schreck, S. (2002). Navier-Stokes predictions of NREL phase VI rotor in the NASA Ames $80 \mathrm{ft} \times 120 \mathrm{ft}$ wind tunnel, Wind Energy, Vol. 5, No. 2-3, pp. 151-169.

Tangler, J.L. \& Kocurek, J.D. (1993). Wind turbine post stall airfoil performance characteristics guidelines for blade-element momentum methods, Proc. of $43^{\text {rd }}$ AIAA Aerospace Sciences Meeting and Exhibit (591), 1-10.

Tangler, J. L. (2002). The nebulous art of using wind tunnel airfoil data for predicting rotor performance, Wind Energy, Vol. 5, pp. 245-257.

Tangler, J.L. (2004). Insight into wind turbine stall and post-stall aerodynamics, Wind Energy, Vol. 7, pp. 247-260.

Vatistas, G.H.; Kozel, V. \& Mih, W.C. (1991). A simpler model for concentrated vortices, Experiments in Fluids, Vol. 11, pp. 73-76. 


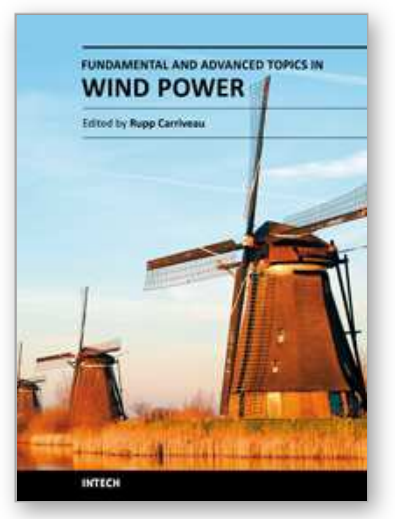

\author{
Fundamental and Advanced Topics in Wind Power \\ Edited by Dr. Rupp Carriveau
}

ISBN 978-953-307-508-2

Hard cover, 422 pages

Publisher InTech

Published online 20, June, 2011

Published in print edition June, 2011

As the fastest growing source of energy in the world, wind has a very important role to play in the global energy mix. This text covers a spectrum of leading edge topics critical to the rapidly evolving wind power industry. The reader is introduced to the fundamentals of wind energy aerodynamics; then essential structural, mechanical, and electrical subjects are discussed. The book is composed of three sections that include the Aerodynamics and Environmental Loading of Wind Turbines, Structural and Electromechanical Elements of Wind Power Conversion, and Wind Turbine Control and System Integration. In addition to the fundamental rudiments illustrated, the reader will be exposed to specialized applied and advanced topics including magnetic suspension bearing systems, structural health monitoring, and the optimized integration of wind power into micro and smart grids.

\title{
How to reference
}

In order to correctly reference this scholarly work, feel free to copy and paste the following:

Horia Dumitrescu and Vladimir Cardos (2011). Inboard Stall Delay Due to Rotation, Fundamental and Advanced Topics in Wind Power, Dr. Rupp Carriveau (Ed.), ISBN: 978-953-307-508-2, InTech, Available from: http://www.intechopen.com/books/fundamental-and-advanced-topics-in-wind-power/inboard-stall-delay-due-torotation

\section{INTECH}

open science | open minds

\author{
InTech Europe \\ University Campus STeP Ri \\ Slavka Krautzeka 83/A \\ 51000 Rijeka, Croatia \\ Phone: +385 (51) 770447 \\ Fax: +385 (51) 686166 \\ www.intechopen.com
}

\author{
InTech China \\ Unit 405, Office Block, Hotel Equatorial Shanghai \\ No.65, Yan An Road (West), Shanghai, 200040, China \\ 中国上海市延安西路65号上海国际贵都大饭店办公楼 405 单元 \\ Phone: +86-21-62489820 \\ Fax: +86-21-62489821
}


(C) 2011 The Author(s). Licensee IntechOpen. This chapter is distributed under the terms of the Creative Commons Attribution-NonCommercialShareAlike-3.0 License, which permits use, distribution and reproduction for non-commercial purposes, provided the original is properly cited and derivative works building on this content are distributed under the same license. 